\title{
Sequential modulations of stimulus-response correspondence effects depend on awareness of response conflict
}

\author{
WILFRIED KUNDE \\ University of Würzburg, Würzburg, Germany
}

\begin{abstract}
In two experiments, sequential modulations of prime-target correspondence effects were investigated in a metacontrast paradigm. Primes were either unmasked and thus consciously discriminable, or entirely masked and thus indiscriminable. Mirroring similar findings from Eriksen- and Simon-type tasks, the influence of prime-target correspondence was reduced in trials that followed a noncorresponding prime-target pair, which suggests that prime-induced response activation can be temporarily suppressed after an incompatible trial. This sequential modulation was independent of prime discriminability in the current trial, but it occurred only when the prime, and thus a conflict between the primeinduced and the deliberately to-be-selected response, was consciously experienced in the preceding trial. This suggests that the suppression of automatic response priming is not an immediate consequence of response conflict, but an intention-mediated strategy.
\end{abstract}

In general, humans possess enormous flexibility in how they react to external stimulation. For example, in a standard choice reaction task (CRT), it usually suffices simply to communicate an arbitrary stimulus-response (S-R) mapping to a participant in order that he or she can follow this instruction virtually error free, given that he or she is willing to do so. Nevertheless, a number of so-called conflict paradigms in experimental psychology have also shown that, subjects' intention notwithstanding, S-R translation cannot be shielded entirely from influences of task-irrelevant information. Typically, these influences are evident in deterioration of performance when task-relevant (i.e., instructed) and task-irrelevant (i.e., to-be-ignored) aspects of a stimulus display provoke different motor responses. For example, in Eriksen-type flanker tasks, response times (RTs) to a target stimulus are longer when the target is concurrently flanked by irrelevant, to-be-ignored stimuli that were mapped onto a different response in preceding trials (Eriksen \& Eriksen, 1974). Likewise, in the Simon paradigm, responses are slower and more error prone when the task-irrelevant, to-be-ignored location of a stimulus does not correspond to the location of the response required by another, task-relevant stimulus attribute (e.g., stimulus color; Simon, 1969).

Findings such as these have motivated the idea that deliberate response selection, which phenomenally appears to

Funding for this research was provided by Grant HO 1301/6-1 of the German Research Foundation. I thank Wolfgang Prinz and Gregory Stevens for their helpful comments on an earlier draft of this paper and Christian Stöcker for improving the English. Correspondence concerning this article should be addressed to W. Kunde, who is now at the Universität Halle-Wittenberg, Institut für Psychologie, 06099 Halle (Saale), Germany (e-mail: w.kunde@psych.uni-halle.de). underlie our reactions to external stimulation, is occasionally bypassed by a more direct, unconditional responseactivation route not under the immediate control of the actor (see, e.g., Kornblum, Hasbroucq, \& Osman, 1990; Neumann \& Klotz, 1994; Zorzi \& Umiltà, 1995). Moreover, there is evidence that this direct or "pragmatic" responsepriming route is neurally distinct from the more slowly working, deliberate response-selection route (see, e.g., Milner \& Goodale, 1995). Correspondence effects of the type described above are assumed to arise when the automatically evoked response is not the one that the stimuli require by instruction, because in this case a response competition emerges that results in an increase of RTs and error rates. ${ }^{1}$

Recently, however, the widely acknowledged assumption of an autonomous response-activation route has been challenged by the observation that correspondence effects are subject to subtle contextual modulations. Sequential analyses of the Eriksen paradigm (see Botvinick, Nystrom, Fissell, Carter, \& Cohen, 1999; Gratton, Coles, \& Donchin, 1992) and of the Simon paradigm (see Hommel, 2000; Kunde \& Stöcker, 2002; Mordkoff, 1998; Praamstra, Kleine, \& Schnitzler, 1999; Stürmer, Leuthold, Soetens, Schröter, \& Sommer, 2002), have revealed that the apparently robust compatibility effects are reduced (and sometimes even absent) when the preceding trial in these conflict paradigms was noncorresponding (e.g., when, in the Simon task, the stimulus appeared on the left side but required a right-sided response task or when, in the Eriksen task, a response-incompatibleflanker was presented). This observation has motivated the idea that the automatic response route can be temporarily blocked after noncorresponding trials, resulting in lower costs of $\mathrm{S}-\mathrm{R}$ noncorrespondence and lower benefits of $\mathrm{S}-\mathrm{R}$ correspondence in 
subsequent trials. I will refer to this idea as the gating account (for an alternative view, see Hommel, 2000).

The gating account suggests that apparently "automatic" response priming is not as unconditional as is commonly assumed, and thus that it provides a challenge for traditional concepts of automaticity, which, by definition, portray automatic processes as being immune to intentional control. It would therefore be desirable to gain a better understanding of the necessary requirements for gating to occur. The present study investigates one such potential requirement: Does gating depend on awareness of a response conflict between automatically primed and deliberately to-be-selected responses, or is it a more or less inevitable consequence of response competition? The former position is in line with the idea of gating as a strategy, determined by the actor's conscious experience and intention. The latter position would suggest that gating is an unmediated, internal adaptation of the system to response competition as such. Existing theories allow no clear decision between these two positions. Although a number of theories assume a responsemonitoring mechanism (e.g., the "verification" process in the dimensional overlap model of Kornblum et al., 1990) and neurophysiological studies point to a plausible neural locus of such a mechanism (presumably in the anterior cingulate cortex; see Botvinick et al., 1999; Carter et al., 1998; Gehring, Goss, Coles, Meyer, \& Donchin, 1993), it is not clear whether the operation of such a response monitoring device is associated with, or affected by, awareness of response conflict.

With a typical Simon or Eriksen paradigm, the dependence/independence of gating on awareness of preceding response conflict is hard to investigate, because the task-irrelevant stimulus features that produce automatic response priming are clearly perceivable, and thus participants are likely to be aware of the competing responses. A straightforward way to address this question is to present the irrelevant stimulus attribute subliminally, so that it still affects performance but remains undetectable for the subjects. This was the rationale behind the present study.

\section{EXPERIMENT 1}

I adopted a prime-target metacontrast paradigm of the type used by Neumann and colleagues (Klotz \& Neumann, 1999; Neumann \& Klotz, 1994; see also Vorberg, Mattler, Heinecke, Schmidt, \& Schwarzbach, in press). The participants' task was to respond to the direction of a target arrow (leftward or rightward; see Figure 1). The target was preceded by a prime that consisted of a smaller replica of the target. In the corresponding condition, the prime pointed in the same direction as did the target, and in the noncorresponding condition it pointed in the opposite direction. In the neutral condition, the prime consisted of two overlapping leftward and rightward arrows. The crucial aspect of the stimuli is that the prime exactly fits within a cutting in the middle of the target, which is known to result in efficient masking (i.e., the primes are not discriminable and are sometimes even undetectable). Nevertheless, the task-irrelevant prime evokes the particular motor response that a corresponding target would require, which results in benefits of corresponding prime-target pairs and costs of noncorresponding pairs $^{2}$ (see, e.g., Leuthold \& Kopp, 1998; Neumann \& Klotz, 1994).

Of particular interest was the comparison of two conditions that will be denoted as the indiscriminable prime condition and the discriminable prime condition. In the indiscriminable prime condition, the prime was efficiently masked (as revealed by postexperimental primediscrimination performance). In the discriminable prime condition, the prime was clearly perceptible, due to a slight increase of presentation duration. The question of interest was whether sequential dependencies of the prime-target correspondence from the preceding trial similar to those observed in other conflict paradigms would emerge, and, provided that they did, whether they

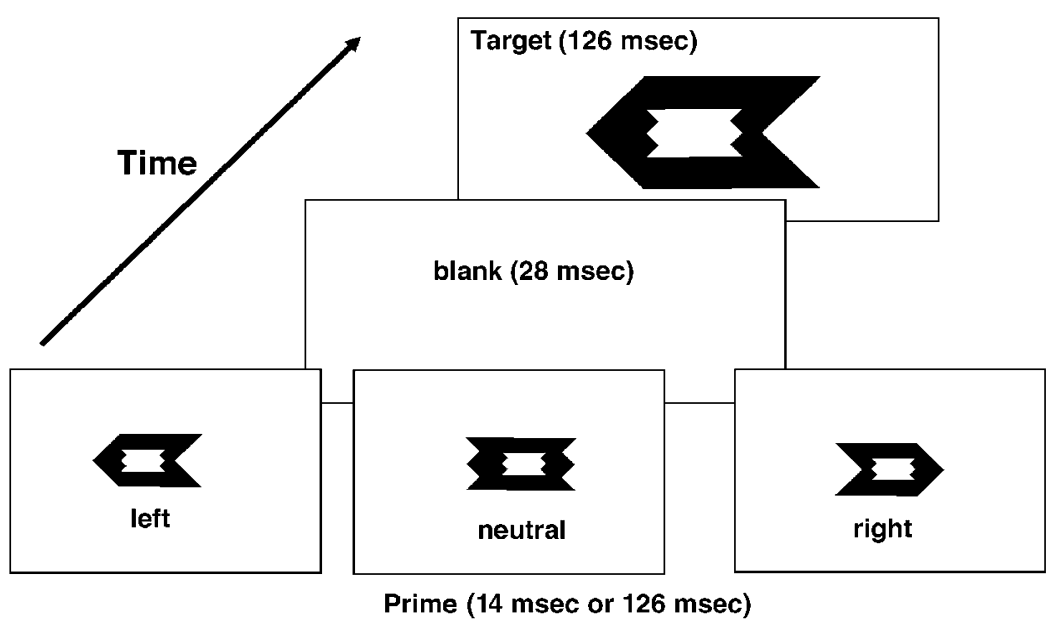

Figure 1. Timeline of an individual trial. 
would depend on the conscious accessibility of this correspondence.

\section{Method}

Participants. Sixteen students (4 men, 12 women) aged 19 to 30 years from the University of Würzburg, with normal or correctedto-normal vision, participated.

Apparatus, Stimuli, and Procedure. An IBM-compatible computer with a 17-in. VGA display was used for stimulus presentation and response sampling. Stimuli were presented in blue on a white background. Viewing distance was approximately $80 \mathrm{~cm}$. The primes were arrows of the type depicted in Figure 1 and they were $15 \mathrm{~mm} \times 10 \mathrm{~mm}$ in size. They were left-pointing, right-pointing, or neutral. Neutral primes resulted from overlap of a left-pointing arrow and a right-pointing arrow. The targets were enlarged leftpointing or right-pointing primes (target:prime ratio of 2.35). The primes fitted exactly into the cutting in the middle of the targets. The participants were instructed to respond to the direction of the target. Their responses were recorded by two microswitches $(12 \times$ $12 \mathrm{~mm}$ ) connected to the parallel port of the computer and positioned centrally in front of the participants. The keys were pressed with the index fingers of the left and right hands.

After a randomly determined intertrial interval of $1,300 \mathrm{msec}$ to $1,600 \mathrm{msec}$, each trial started with an auditory $20-\mathrm{msec}$ warning click $(100 \mathrm{~Hz})$. The prime was presented $750 \mathrm{msec}$ after click onset, and it was followed by a blank interval of $28 \mathrm{msec}$. Then the target was presented for $126 \mathrm{msec}$. The participants were to respond on the side to which the target pointed. For the eight participants in the indiscriminable prime condition, the prime was presented for $14 \mathrm{msec}$, whereas it was presented for $126 \mathrm{msec}$ for the eight participants in the discriminable prime condition. After 108 practice trials, the participants performed 540 trials, of which 240 were prime-target corresponding, 240 were prime-target noncorresponding, and 60 were neutral. For each participant, a new random order of trials was generated.

Following these experimental trials, the participants performed a prime-discrimination task and underwent an extensive postexperimental interview. They were informed about the presence and possible forms of the primes and were instructed to decide at leisure if the prime presented was left-pointing, right-pointing, or neutral. The participants made these judgments by pressing one of three keys of the computer keyboard in a forced-choice situation. The participants were presented with 24 left-pointing, 24 right-pointing, and 24 neutral primes, followed equally frequently by either a left-pointing or a right-pointing target as in the experimental trials. Error feedback was provided only at the end of the discrimination task. After the discrimination task, the participants were asked if they had noticed the presence and correspondence/ noncorrespondence of primes and targets in the experimental trials, and how they had handled it.

\section{Results}

Prime-discrimination task. The average proportion of correct prime discriminations was $32.3 \%$ at the prime duration of $14 \mathrm{msec}$, which is not significantly different from the chance level of $33 \%(F<1)$. The maximum hit rate of an individual participant was $37.5 \%$. In contrast, at the prime duration of $126 \mathrm{msec}$, the percentage of correct prime discriminations was $87.2 \%$, which is significantly above chance $(p<.01)$. Thus, as intended, the primes were entirely indiscriminable at the duration of $14 \mathrm{msec}$, whereas they were clearly discriminable at the duration of $126 \mathrm{msec}$.

Experimental trials. RTs from correct responses were entered into an analysis of variance (ANOVA) with the between-subjects variable of prime discriminability (discriminable vs. indiscriminable) and the within-subjects variables of prime-target correspondence (corresponding, neutral, or noncorresponding) and prime-target correspondence in the preceding trial (also corresponding, neutral, or noncorresponding). The mean RTs and error rates from the factorial combinations of these variables are listed in Table 1.

The analysis revealed a significant influence of primetarget correspondence $[F(2,28)=83.72, p<.01]$. RTs with a corresponding prime were shorter than those with a neutral prime, which in turn were shorter than those with a noncorresponding prime. The influence of prime-target correspondence interacted with that of the prime-target correspondence in the preceding trial $[F(4,56)=4.14$, $p<.01]$.

Figure 2 depicts the size of the correspondence effect (noncorresponding-corresponding) as a function of the correspondence in the preceding trial. With a noncorresponding trial preceding, the prime-target correspondence in the current trial had an overall weaker impact than it did with a corresponding trial preceding. However, this was entirely due to the condition with discriminable primes (i.e., pries of $126-\mathrm{msec}$ duration), resulting in a

Table 1

Experiment 1: Mean Response Times (in Milliseconds) and Error Rates (in Percentages) as a Function of Prime-Target Correspondence, Prime-Target Correspondence in the Preceding Trial, and Discriminability of the Prime

\begin{tabular}{|c|c|c|c|c|c|c|}
\hline \multirow[b]{3}{*}{ Trial $n-1$} & \multicolumn{6}{|c|}{ Prime-Target Correspondence in Trial $n$} \\
\hline & \multicolumn{2}{|c|}{ Noncorresponding } & \multicolumn{2}{|c|}{ Neutral } & \multicolumn{2}{|c|}{ Corresponding } \\
\hline & RT & PE & RT & PE & RT & $\mathrm{PE}$ \\
\hline \multicolumn{7}{|l|}{ Indiscriminable primes } \\
\hline Noncorresponding & 380 & 4.6 & 334 & 1.7 & 316 & 0.1 \\
\hline Neutral & 381 & 7.4 & 316 & 0.0 & 310 & 1.3 \\
\hline Corresponding & 375 & 6.3 & 332 & 2.1 & 313 & 0.5 \\
\hline \multicolumn{7}{|l|}{ Discriminable primes } \\
\hline Noncorresponding & 392 & 1.6 & 375 & 0.0 & 344 & 0.1 \\
\hline Neutral & 396 & 5.8 & 378 & 3.1 & 324 & 0.5 \\
\hline Corresponding & 404 & 8.4 & 369 & 0.5 & 317 & 0.1 \\
\hline
\end{tabular}

Note-RT, response times; PE, percentage of errors. 

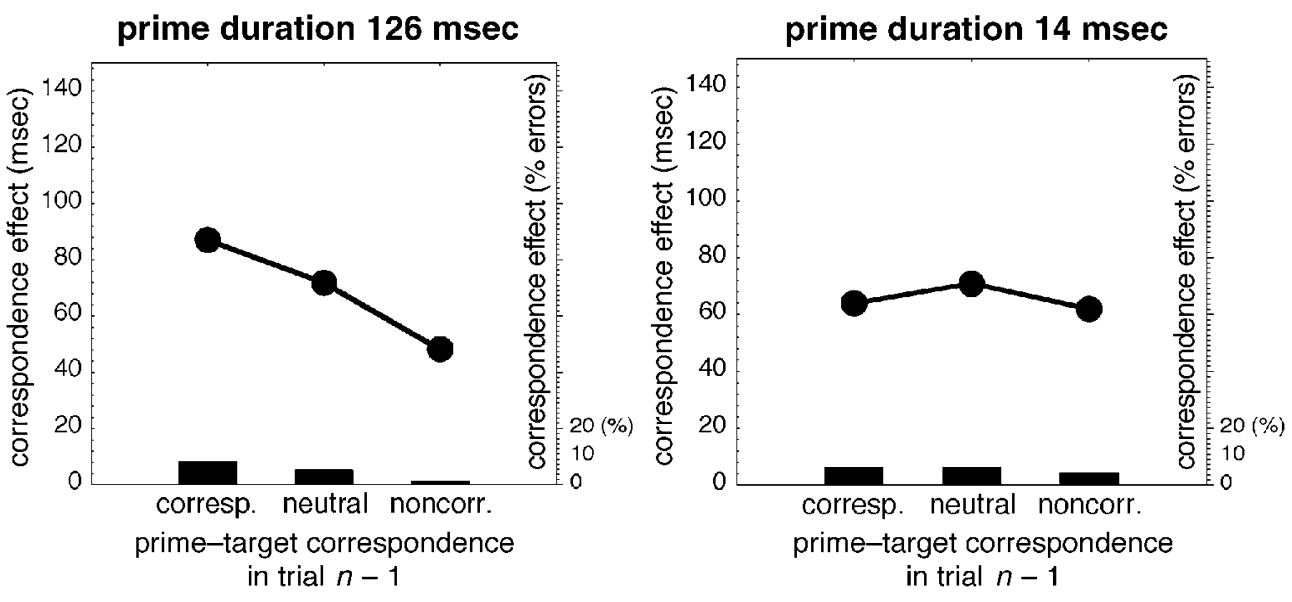

Figure 2. Experiment 1: Correspondence effect (noncorresponding-corresponding) as a function of correspondence in trial $n-1$ and prime duration.

triple interaction of prime discriminability, prime-target correspondence, and prime-target correspondence in the preceding trial $[F(4,56)=5.30, p<.01]$. Single comparisons revealed a highly reliable influence of prime-target correspondence in the preceding trial with discriminable primes $[F(4,28)=7.50, p<.001]$, but no modulating influence with indiscriminable primes $(F<1)$. The only additional source of variance was the interaction between prime discriminability and primetarget correspondence $[F(2,28)=4.11, p<.05]$. This interaction results from the fact that RTs with neutral primes were longer with discriminable than with indiscriminable primes. It is likely that this reflects a frequency effect. Neutral primes were less frequent than left-pointing or right-pointing primes (since they were not of particular interest in the present study), and this may have led to some kind of unspecific distraction when subjects experienced such a relatively rare stimulus in the case that the primes were perceptible, whereas this distraction could not have taken place when the primes were imperceptible. (This interpretation is corroborated by Experiment 2, in which equiprobable neutral primes yielded similar RTs independent of their discriminability.)

The error analysis replicated the influence of primetarget correspondence. In addition, error rates were lower when a noncorresponding trial preceded $[F(2,28)=$ $6.58, p<.01]$. However, this influence was virtually exclusively due to the reduction of error rates with noncorrespondence in the preceding trial (suggesting a blocking of the automatic route) in the group with discriminable primes, resulting in a marginally significant three-way interaction $[F(4,56)=2.26, p<.08]$.

Postexperimental interview. No participant from the indiscriminable-prime condition noticed the correspondence or noncorrespondence of the primes and the targets, and no participant reported that he or she had adopted a strategy that somehow made use of the prime-target re- lation. In contrast, 7 of the 8 subjects in the group with discriminable primes reported that they had noticed the correspondence/noncorrespondence of primes and targets, and that they had found noncorrespondence "inconvenient," because in these cases they had had to "reprogram" their responses. Some of them also said that they occasionally were more "suspicious" after having experienced a noncorresponding prime-target pair. Nevertheless, they they could not clearly articulate their strategy for coping with prime-target noncorrespondence.

\section{Discussion}

Experiment 1 revealed a similar reduction of correspondence effect after noncorresponding trials, as has recently been reported for other conflict paradigms. More importantly, however, this sequential modulation was present only when the source of response conflict (the prime) was clearly perceptible, which suggests that conscious experience of a preceding response conflict is a necessary precondition for these sequential modulations to occur.

However, prime discriminability was varied between subjects in Experiment 1 and, thus, primes were either discriminable or indiscriminable in both the preceding trial and the current trial. It is therefore indeterminable whether experience of response conflict in the preceding trial and/or in the current trial is crucial. One could, for example, argue that a reduction of prime-induced response activation is simply based on perceptual blocking of the prime, which would likely require some perceptual accessibility of the prime at the time when the suppression is required (i.e., in the current trial). In Experiment 2 , trials with discriminable and indiscriminable primes were randomly mixed. If gating depends on experience of previous rather than current response conflict, we should see a modulation of correspondence effects with a discriminable prime in the preceding trial even when the prime in the current trial is indiscriminable, whereas we should not find sequential modula- 
tions when the preceding trial contained an indiscriminable prime even when the current prime is discriminable. Assuming that experience of previous response conflict is crucial, another related prediction is tenable: The intersperse of indiscriminable primes (presumably preventing experience of response conflict) should increase the overall correspondence effect in comparison with the discriminable-prime group of Experiment 1, whereas the intersperse of discriminable primes (and thus the experience of response conflicts) should decrease the overall correspondence effect in comparison with the indiscriminable-prime group, which never experienced any response conflict. Observing this effect would imply that there is some carryover of detected response conflict beyond the immediately following trial.

\section{EXPERIMENT 2}

\section{Method}

Twelve students ( 2 men and 10 women) 20-26 years of age from the University of Würzburg participated. Experiment 2 was a replication of Experiment 1, with the two modifications that primes with a duration of $14 \mathrm{msec}$ and $126 \mathrm{msec}$ were mixed randomly trial by trial, and that left-pointing, right-pointing, and neutral primes were equiprobable. The participants performed five blocks of 144 trials each. The first block was considered practice.

\section{Results}

Prime-discrimination task. The average proportion of correct prime discriminations was $37.7 \%$ at the prime duration of $14 \mathrm{msec}$, which is slightly, though not significantly, different from chance level (33.3\%). The relatively high hit rate is due primarily to two subjects with individual hit rates of $44 \%$. However, no aspect of the data pattern reported in the following is affected when these subjects are excluded from the analysis. At the prime duration of $126 \mathrm{msec}$, the percentage of correct prime discriminations was $83.5 \%$, which well exceeds chance $(p<.01)$.

Experimental trials. RTs from correct responses were entered into an ANOVA with the within-subjects variables of prime discriminability, prime-target corre- spondence, prime discriminability in trial $n-1$, and prime-target correspondence in trial $n-1$. The mean RTs and error rates from the factorial combinations of these variables are listed in Table 2.

There was a pronounced influence of prime-target correspondence: Overall, RTs were $77 \mathrm{msec}$ higher with a noncorresponding than with a corresponding primetarget pair $[F(2,22)=243.23, p<.01]$. In addition, overall, responding was $10 \mathrm{msec}$ slower with a discriminable than with an indiscriminable prime in the preceding trial $[F(1,11)=20.50, p<.01]$ and $8 \mathrm{msec}$ slower with a noncorresponding than with a corresponding prime in the preceding trial $[F(2,22)=11.32, p<.01]$.

Most interesting in the present context is the observation that the prime-target correspondence effect was modified by the correspondence in the preceding trial $[F(4,44)=5.23, p<.01]$. The size of the correspondence effect as a function of correspondence in trial $n-1$ for the four possible successions of prime duration in trial $n$ and in trial $n-1$ is shown in Figure 3.

As predicted, a sequential modulation of correspondence effects was present when the preceding trial contained a discriminable prime (see the left panels in Figure 3) but not when it contained an indiscriminable prime, irrespective of prime discriminability in the current trial (see the right panels of Figure 3), resulting in a significant interaction of correspondence, correspondence in trial $n-1$, and prime duration in trial $n-1$ $[F(4,44)=5.32, p<.01]$. Single comparisons revealed significant influences of correspondence in trial $n-1$ in both conditions in which trial $n-1$ contained a discriminable prime (both $p$ s <.02). The size of this influence (i.e., the slopes in the left panels of Figure 3 ) did not vary significantly $(F<1)$. No influence of correspondence from trial $n-1$ was present when trial $n-1$ contained an indiscriminable prime (both $p$ s $>.15$ ).

The correspondence effect was larger with the prime duration of $126 \mathrm{msec}$ than with that of $14 \mathrm{msec}[F(2,22)=$ $64.98, p<.01]$, particularly when an indiscriminableprime was used in the preceding trial $[F(2,22)=5.60, p<.02$ for the correspondence $\times$ current prime duration $\times$ preceding

Table 2

Experiment 2: Mean Response Times (in Milliseconds) and Error Rates (in Percentages) as a Function of Prime Discriminability and Prime-Target Correspondence in Trial $n$ and Trial $n-1$

\begin{tabular}{|c|c|c|c|c|c|c|c|c|c|c|c|c|}
\hline \multirow[b]{4}{*}{ Trial $n-1$} & \multicolumn{12}{|c|}{ Trial $n$} \\
\hline & \multicolumn{6}{|c|}{ Indiscriminable Prime } & \multicolumn{6}{|c|}{ Discriminable Prime } \\
\hline & \multicolumn{2}{|c|}{ Noncorresponding } & \multicolumn{2}{|c|}{ Neutral } & \multicolumn{2}{|c|}{$\overline{\text { Corresponding }}$} & \multicolumn{2}{|c|}{ Noncorresponding } & \multicolumn{2}{|c|}{ Neutral } & \multicolumn{2}{|c|}{ Corresponding } \\
\hline & RT & $\mathrm{PE}$ & RT & $\overline{\mathrm{PE}}$ & RT & $\mathrm{PE}$ & RT & $\mathrm{PE}$ & $\overline{\mathrm{RT}}$ & $\overline{\mathrm{PE}}$ & RT & $\mathrm{PE}$ \\
\hline \multicolumn{13}{|l|}{ Indiscriminable prime } \\
\hline Noncorresponding & 381 & 1.0 & 350 & 0.0 & 336 & 1.1 & 432 & 23.3 & 354 & 2.1 & 298 & 0.5 \\
\hline Neutral & 374 & 1.0 & 354 & 1.5 & 339 & 0.6 & 415 & 18.2 & 353 & 2.6 & 293 & 0.0 \\
\hline Corresponding & 381 & 2.6 & 344 & 0.0 & 335 & 0.0 & 430 & 26.5 & 355 & 2.0 & 288 & 0.0 \\
\hline \multicolumn{13}{|l|}{ Discriminable prime } \\
\hline Noncorresponding & 381 & 0.4 & 367 & 0.0 & 362 & 0.0 & 421 & 5.7 & 377 & 0.0 & 339 & 0.0 \\
\hline Neutral & 384 & 0.5 & 362 & 0.0 & 351 & 0.0 & 414 & 16.9 & 366 & 0.5 & 312 & 0.0 \\
\hline Corresponding & 386 & 0.5 & 356 & 0.9 & 340 & 0.0 & 420 & 22.8 & 362 & 1.6 & 294 & 0.0 \\
\hline
\end{tabular}

Note-RT, response times; PE, percentage of errors. 

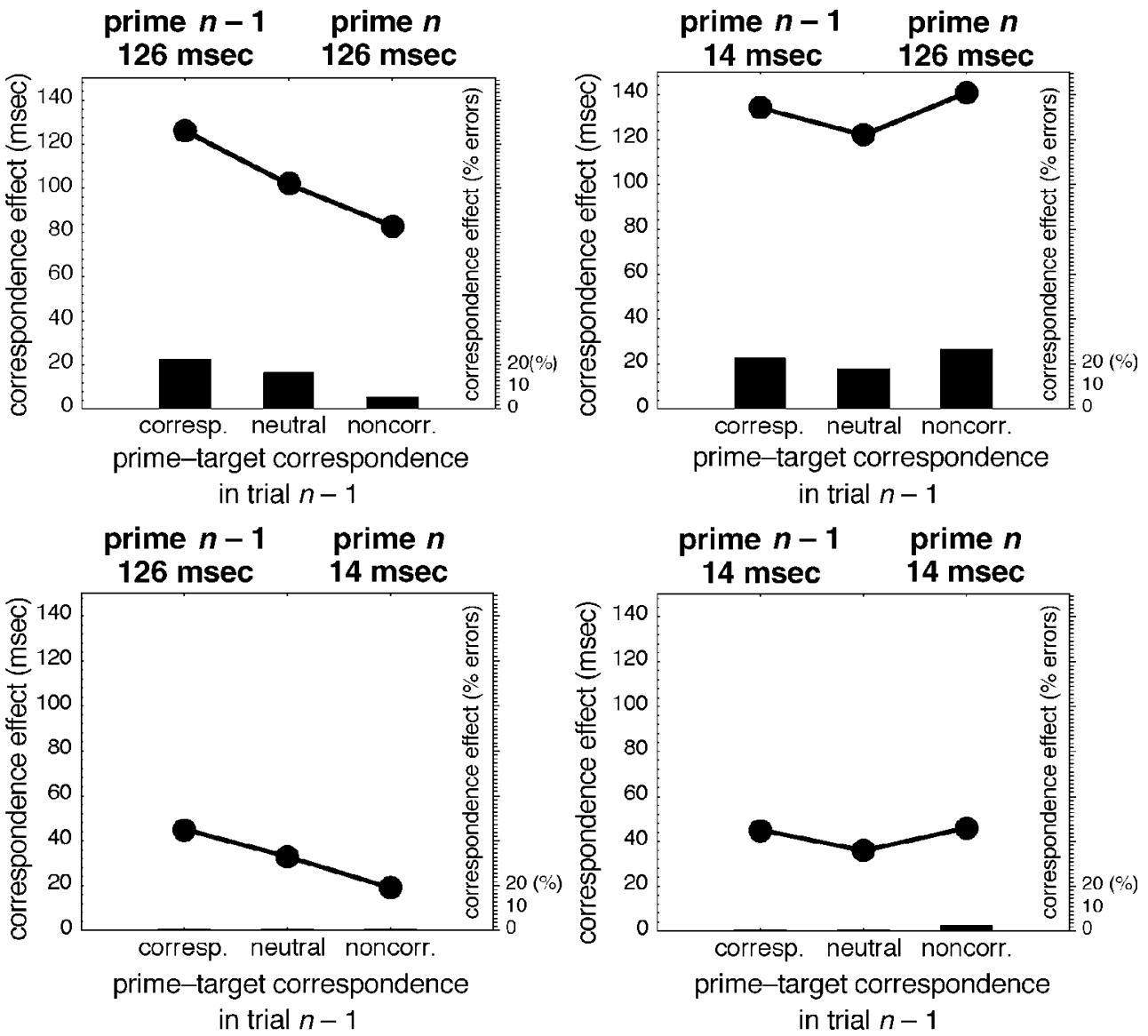

Figure 3. Experiment 2: Correspondence effect (noncorresponding-corresponding) as a function of prime duration in trial $n-1$, prime duration in trial $n$, and correspondence in trial $n-1$.

prime duration interaction]. A comparison with Experiment 1 also shows that, as expected, the correspondence effect with the 126 -msec prime duration was greater, whereas it was smaller with a prime duration of $14 \mathrm{msec}$. Finally, higher RTs with a noncorresponding than with a corresponding prime in the preceding trial were present primarily when the prime in the preceding trial was discriminable, producing an interaction between correspondence in trial $n-1$ and prime discriminability in trial $n-1[F(2,22)=5.14, p<.02]$.

The mean error rate was low $(3.7 \%)$. Errors were practically confined to the trials with a discriminable noncorresponding prime (see Table 2). The absence of errors in virtually half of the conditions allowed no reasonable parametric analysis. However, RTs and error rates were positively correlated for each individual subject, ruling out the possible contribution of speed-accuracy tradeoffs.

\section{GENERAL DISCUSSION}

The present study led to three major outcomes. First, a reduction of correspondence effects following noncor- responding $\mathrm{S}-\mathrm{R}$ events, observed in Simon-type and Eriksen-type conflict tasks, also manifests itself in the prime-target paradigm used in the present study. Correspondence effects in these paradigms are known to result, at least predominantly, from a response conflict between automatic response activation and controlled response selection (see, e.g., Kornblum et al., 1990; Leuthold \& Kopp, 1998; Neumann \& Klotz, 1994). It is therefore reasonable to assume that sequential modulations of correspondence effects in these paradigms can be traced back to the same mechanism-that is, the gating of automatic response priming.

Second, a sequential modulation of the correspondence effect was evident only when, in the preceding trial, the sources of the response conflict (primes and target), and thus the response conflict itself, were clearly perceptible. This suggests that the gating of automatic response priming is not an unmediated consequence (or aftereffect) of any existing response competition as such, but a strategic attempt of the participants to suppress automatic response priming. Moreover, at least in Experiment 2 , responding was overall slightly slower when the 
preceding trial contained a visible prime. Thus, it appears as though subjects entered into a more cautious response mode when they identified a potential source of response conflict in the preceding trial, and particularly so when this source actually produced response conflict.

Third, although conscious prime perception in the preceding trial was crucial for sequential effects to occur, conscious perception of the prime in the current trial was not. This observation has some theoretical implications for the functional locus of gating. Viewed from traditional stage theory, the present variation of prime discriminability (ranging from virtually complete discriminability to complete indiscriminability)represents a massive variation of a factor affecting perceptual stages of information processing. Yet, the sequential modulation of the correspondence effect was relatively independent of prime discriminability in the current trial. Following traditional additive factors logic (Sternberg, 1969), this suggests that the sequential modulation has its origin in somewhat later, postperceptual stages of processing.

In accordance with a postperceptual locus, Stürmer and Leuthold (1998) found a dissociation between ERPs over motor areas and over posterior parietal cortex (PPC) as a function of the correspondence sequence in a Simon task. Whereas activation of the PPC, which is known to mediate sensorimotor coding (see, e.g., Andersen, Snyder, Bradley, \& Xing, 1997; Milner \& Goodale, 1995), was present independently of whether the preceding trial was corresponding or noncorresponding, an incorrect activation over motor areas, indicating automatic response priming, was present exclusively following corresponding trials (indicating suppression of response activation in the case of preceding noncorrespondence). Thus, it appears that sensorimotor information is blocked along its way from PPC to primary motor cortex. This observation is in line with the idea that only the initial stimulus-related aspect of processing of the prime is inevitable and, thus, automatic in a strict sense, but that further prime processing can be offset by a voluntary attempt to do so. However, because the prime-target correspondence effect may result, at least to a certain degree, from perceptual processes (see note 2), and because it was not entirely eliminated in the present study, the functional locus of gating deserves further investigation.

The dependence of sequential modulations on awareness of a response conflict, found in the present study, may account for some recent failures to observe such modulations. For example, Kunde and Stöcker (2002) found no influence of preceding correspondence in a nonspatial variant of the Simon effect. They had subjects press a key either briefly or longer in response to the color of a stimulus that also varied along its task-irrelevant presentation duration ( $42 \mathrm{msec}$ or $200 \mathrm{msec}$ ). A robust influence of correspondence between irrelevant stimulus duration and response duration was observed, which, however, was unaffected by the S-R duration correspondence in the preceding trial. Given the present findings, it seems possible that although the noncorrespon- dence between stimulus duration and response duration affected performance, it was not consciously perceived. Likewise, Kornblum and Stevens (2002) reported a failure to observe sequential modulation of correspondence effects in a four-choice reaction task. Subjects responded to a centrally presented letter by moving a joystick up, down, left, or right. Irrelevant arrows pointing up, down, left, or right were presented as flankers. The authors used a four-choice reaction to avoid confounding s potentially present in two-choice tasks, and thus concluded that the absence of sequential effects in their four-choice task points to the causal role of these confounded factors (such as repetition/nonrepetition of relevant and irrelevant stimuli). ${ }^{3}$ Apart from other reasons for this failure, it also seems tenable that, due to the high attentional focus on the central target letter, the response conflict emerging from the laterally presented arrows was not experienced sufficiently clearly, although it still produced correspondence effects. This conjecture requires additional support.

The present study broadens the validity of the gating account motivated by studies of irrelevant S-R compatibility to a quite different experimental situation. Furthermore, it shows that conscious experience of response conflict is a precondition for gating to occur. This provides evidence for the conjecture that "automatic" $\mathrm{S}-\mathrm{R}$ translation may not be as immune to strategic influences, and hence not as qualitatively different from "controlled" $\mathrm{S}-\mathrm{R}$ translation, as has traditionally been assumed.

\section{REFERENCES}

Andersen, R. A., Snyder, L. H., Bradley, D. C., \& Xing, J. (1997). Multimodal representation of space in the posterior parietal cortex and its use in planning movements. Annual Review of Neuroscience, 20, 303-330.

Botvinick, M., Nystrom, L., Fissell, K., Carter, C., \& Cohen, J. (1999). Conflict monitoring versus selection-for-action in anterior cingulated cortex. Nature, 402, 179-181.

Carter, C. S., Braver, T. S., Barch, D. M., Botvinick, M. M., Noll, D., \& Cohen, J. D. (1998). Anterior cingulate cortex, error detection, and the online monitoring of performance. Science, $\mathbf{2 8 0}$, 747-749.

Coles, M. G. H., Gratton, G., Bashore, T. T., Eriksen, C. W., \& Donchin, E. (1985). A psychophysiologicalinvestigation of the continuous flow model of human information processing. Journal of Experimental Psychology: Human Perception \& Performance, 11, 529553.

De Jong, R., Liang, C. C., \& Lauber, E. (1994). Conditional and unconditional automaticity: A dual-process model of effects of spatial stimulus-response correspondence. Journal of Experimental Psychology: Human Perception \& Performance, 20, 731-750.

Eriksen, B. A., \& Eriksen, C. W. (1974). Effects of noise letters upon the identification of a target letter in a nonsearch task. Perception \& Psychophysics, 16, 143-149.

Gehring, W. J., Goss, B., Coles, M. H. G., Meyer, D. E., \& DonCHIN, E. (1993). A neural system for error detection and compensation. Psychological Science, 4, 385-390.

Gratton, G., Coles, J., \& Donchin, E. (1992). Optimizing the use of information: Strategic control of activation of responses. Journal of Experimental Psychology: General, 121, 480-506.

Hommes, B. (1997). Interactions between stimulus-stimulus congruence and stimulus-response compatibility. Psychological Research, 59, 248-260. 
Hommel, B. (2000). A feature integration account of sequential effects in the Simon task. Manuscript submitted for publication.

Klotz, W., \& Neumann, O. (1999). Motor activation without conscious discrimination in metacontrast masking. Journal of Experimental Psychology: Human Perception \& Performance, 25, 976-992.

KLOTZ, W., \& WolfF, P. (1995). The effect of a masked stimulus on the response to the masking stimulus. Psychological Research, 58, 92-101.

Kornblum, S., HasbroucQ, T., \& Osman, A. (1990). Dimensional overlap: Cognitive basis for stimulus response compatibility: A model and taxonomy. Psychological Review, 97, 253-270.

Kornblum, S., \& Stevens, G. (2002). Intertrial effects of dimensional overlap: Findings and issues. In W. Prinz \& B. Hommel (Eds.), Common mechanisms in perception and action: Attention and performance XIX (pp. 9-54). Cambridge, MA: MIT Press.

Kunde, W., \& STÖcKer, C. (2002). A Simon effect for stimulus-response duration. Quarterly Journal of Experimental Psychology. Section A: Human Experimental Psychology, 55A, 581-582.

Leuthold, H., \& Kopp, B. (1998). Mechanisms of priming by masked stimuli: Inferences from event-related brain potentials. Psychological Science, 9, 263-269.

Milner, A. D., \& Goodale, M. A. (1995). The visual brain in action. New York: Oxford University Press.

MordKoff, T. (1998). The gating of irrelevant information in selectiveattention tasks. Abstracts of the Psychonomic Society, 3, 21.

Neumann, O., \& KLOTz, W. (1994). Motor responses to nonreportable, masked stimuli: Where is the limit of direct parameter specification? In C. Umiltà \& M. Moscovitch (Eds.), Attention and performance $X V$ : Conscious and nonconscious information processing (pp. 123150). Cambridge, MA: MIT Press.

Praamstra, R. Kleine, B. U., \& Schnitzler, A. (1999). Magnetic stimulation of the dorsal premotor cortex modulates the Simon effect. NeuroReport, 10, 3671-3674.

Simon, J. R. (1969). Reactions toward the source of stimulation. Journal of Experimental Psychology, 81, 174-176.

SoETENS, E. (1998). Localizing sequential effects in serial choice reaction time with the information reduction procedure. Journal of Experimental Psychology: Human Perception \& Performance, 24, 547-568.

STERnBERG, S. (1969). The recovery of processing stages: Extensions of Donders' method. Acta Psychologica, 30, 276-315.

Stürmer, B., \& Leuthold, H. (1998, July). Functional dissociation of human posterior parietal cortex and frontal motor cortex in visuomotor priming. Poster presented at the 12 th International Conference on Event-Related Potentials of the Brain, Boston.

Stürmer, B., Leuthold, H., Soetens, E., Schröter, H., \& SomMER, W. (2002). Control over location-based response activation in the Simon task. Journal of Experimental Psychology: Human Perception \& Performance, 28, 1345-1363.

Vorberg, D., Mattler, U., Heinecke, A., Schmidt, T., \& Schwarz$\mathrm{BACH}, \mathrm{J}$. (in press). Invariant time-course of priming with and without awareness. In C. Kaernbach, E. Schröger, \& H. Müller (Eds.), Psychophysics beyond sensation: Laws and invariants of human cognition. Mahwah, NJ: Erlbaum.

ZHANG, J., \& Kornblum, S. (1998). The effects of stimulus-response mapping and irrelevant stimulus-response and stimulus-stimulus overlap in four-choice Stroop tasks with single-carrier stimuli. Journal of Experimental Psychology: Human Perception \& Performance, 24, 3-19.

ZoRZI, M., \& UMiLtà, C. (1995). A computational model of the Simon effect. Psychological Research/Psychologische Forschung, 58, 193-205.

\section{NOTES}

1. Although there is an ongoing debate on the functional locus of the various correspondence effects in conflict paradigms (see, e.g., Hommel, 1997; Zhang \& Kornblum, 1998) there is convincing electrophysiological evidence for automatic activation of incorrect responses in incompatible trials in the Eriksen task (see, e.g., Coles, Gratton, Bashore, Eriksen, \& Donchin, 1985), the Simon task (see, e.g., De Jong, Liang, \& Lauber, 1994), and the metacontrast paradigm employed in the present study (Leuthold \& Kopp, 1998). Thus, it is fair to conclude that automatic response activation is at least a functional commonality of all these paradigms.

2. The present version of the task can be described as a kind of spatial Stroop task, because the relevant target, the irrelevant prime, and the responses refer to the same spatial concepts (left and right). Thus, in principle, the prime-target correspondence effect could originate from (1) the inherent direction information of the prime, which may automatically induce a spatially corresponding response, (2) the physical similarity between prime and target, allowing for faster perceptual analysis of a prime-corresponding target, or (3) the fact that the prime evokes the particular response to which a corresponding target is mapped. Previous studies suggest, however, that the former two influences are of minor relevance. Neumann and Klotz (1994) and Klotz and Wolff (1995) found correspondence effects numerically very similar to those in the present study, with nonspatial geometrical stimuli, which makes an explanation in terms of spatial overlap between primes and responses unlikely. They also demonstrated that physically dissimilar primes produce correspondence effects, provided that they match any response-relevant target (whether or not it is presented in the same trial as the prime), ruling out a pure perceptual priming account. Leuthold and Kopp (1998) found prime-induced response activation in premotor areas by means of the lateralized readiness potential. In sum, these findings suggest that metacontrast priming effects originate from direct activation of prime-associated motor responses (which Neumann \& Klotz, 1994, call "direct parameter specification"; see also the General Discussion section of the present article).

3. I looked for an impact of these factors in the present study. If they had an influence, one would expect considerable performance differences within a given type of correspondence repetition, depending on whether the relevant or the irrelevant stimulus is repeated. For example, the prime-target pair $>>$ should yield markedly superior performance when followed by the prime-target pair $>>$ than when followed by the pair $<<$, which, however, is not the case (314 msec vs. $315 \mathrm{msec}$ in Experiment 2). In other words, trial-to-trial repetitions of relevant and irrelevant stimuli (i.e., primes and targets) were relatively ineffective and, given the relatively long response-stimulus interval (of at least $2,000 \mathrm{msec}$ ), there is little reason to expect them (see Soetens, 1998, for a review). Moreover, one would have to assume that the suspected confoundings were effective in those conditions that yielded sequential effects (e.g., when prime $n-1$ was discriminable but prime $n$ was not) but ineffective in those conditions that yielded no effects (e.g., when prime $n-1$ was not discriminable but prime $n$ was), which, in my opinion, is a relatively implausible assumption.

(Manuscript received February 21, 2001; revision accepted for publication December 17, 2001.) 